UNITED STATES

DEPARTMENT OF THE INTERIOR

GEOLOGICAL SURVEY

DISCHARGE, GAGE HEIGHT, AND ELEVATION OF

100-YEAR FLOODS IN THE HUDSON RIVER BASIN

By Roger $J$. Archer

U.S. GEOLOGICAL SURVEY

Open-File Report $78-332$

Prepared in cooperation with the U.S. Water Resources Council

for the Hudson River Basin Study Group

Albany, New York

April 1978 
CONTENTS

Page

Conversion factors and abbreviations................. 11

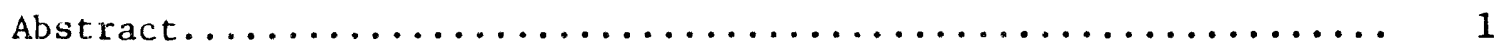

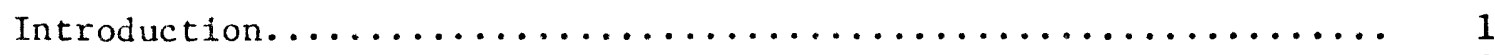

Data computation and presentation...................... 2

References cited................................ 2

\section{ILLUSTRATIONS \\ (Plate is in pocket)}

Plate 1. Map of Hudson River basin showing location of gaging stations for which discharge of 100-year flood is available.

\section{TABLES}

Table 1. Discharge, gage height, and elevation of 100-year

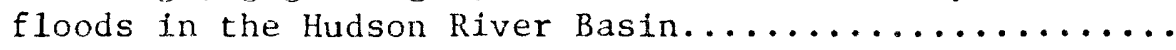

FACTORS FOR CONVERTING U.S. CUSTOMARY UNITS TO

INTERNATIONAL SYSTEM (SI) UNITS

Multiply U.S. Customary units

feet ( $f t$ )

square miles $\left(\mathrm{mi}^{2}\right)$

cubic feet per second

$\left(\mathrm{ft}^{3} / \mathrm{s}\right)$
By

0.3048

2.59

.028317
To obtain SI units

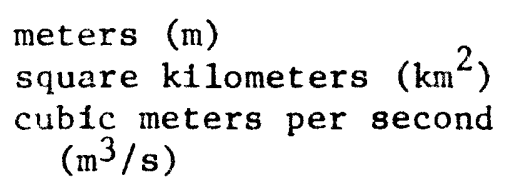
cubic meters per second $\left(\mathrm{m}^{3} / \mathrm{s}\right)$ 


\title{
DISCHARGE, GAGE HEIGHT, AND ELEVATION OF \\ 100-YEAR FLOODS IN THE HUDSON RIVER BASIN
}

By

Roger J. Archer

\begin{abstract}
The flood discharge that may be expected to be equaled or exceeded on the average of once in a 100 years (100-year flood) was computed by use of the log-Pearson Type III frequency distribution for 72 stations in the Hudson River basin. These discharges and, where avallable, their corresponding gage height and elevation above mean sea level, are presented in tabular form. A short explanation of computation methods is included. The data are to be used as part of a Federally funded study of the water resources and related land resources of the Hudson River basin.
\end{abstract}

\section{INTRODUCTION}

Drought during the early 1960's provided a stimulus for studies by several agencies on water-resources planning and management in New York State. From the results of these studies, the State of New York prepared a proposal for a "Level B Study of the Hudson River basin," designed to evaluate present and future water and related land resources of the Hudson River basin. That study was funded by Congress through the Water Resources Council (Hudson River Basin Study Group, written commun., Nov. 1976, p. 1). The New York State Department of Environmental Conservation was selected to provide guidance for the study.

Work groups composed of representatives from selected State and Federal agencies, including the U.S. Geological Survey, were formed to assemble needed data for the Level B study. As one phase of its part in the study, the Survey was asked to provide a summary of information on the discharge, gage height, and elevation of the "100-year flood" at all locations in the Hudson River basin for which this information could be obtained. The term 100-year flood designates the discharge that can be expected to be equaled or exceeded once in 100 years on the average, or the discharge that has a 1-percent chance of being exceeded in any given year [U.S. Water Resources Council, 1976, p. 22]. This flood information is to be used by the Flood Damage Reduction work group in their phase of the Level $B$ study. 


\section{Data Computation and Presentation}

The 100-year flood discharge at all continuous-gaging and partialrecord crest-stage stations in the Hudson River basin with at least 10 consecutive years of record through 1975 was computed by the log-Pearson Type III method of determining the frequency relationship. These data are given in table 1 ; locations of the stations are shown in plate 1. Also included in table 1 are gage height and elevations of the 100-year flood at the gaging stations and gage heights at the crest-stage stations.

The 100-year flood discharges were computed in accordance with "Guidelines for Determining Flood Flow Frequency," Bulletin 17 of the Hydrology Committee, U.S. Water Resources Counc1l (1976). The guldelines allow for several options, of which the following were used in the computations: (1) Only the maximum flood peak in each water year was considered; (2) the skew coefficients used were the generalized values obtained from the skew map in U.S. Water Resources Council Bulletin 17 (1976); (3) the 100-year floods were not adjusted for expected probability; and (4) where appropriate, historic flood information was used to adjust the frequency curve.

U.S. Water Resources Council Bulletin 17 warns users of floodfrequency data to be aware that such data are only estimates from the population curves based on log Pearson Type III frequency relationship and are not exact representations. The streamflow records on which these estimates are based are only a sampling; how well the sampling will represent the total flood experience (past, present, and future) depends upon the number of years of record and representiveness of the recorded flood peaks.

The guidelines are applicable unly to naturally flowing, unregulated streams. Computations for regulated streams (those receiving regulated flow from more than 20 percent of the drainage area) were based on observed station skew rather than generalized skew. Frequency relationships of regulated streams were computed only for those stations having a minimum of 25 years of record subsequent to start of regulation. Changes in operational patterns can change flood-frequency relationships downst ream.

\section{REFERENCES CITED}

Hudson River Basin Study Group, 1976, Amended plan of study, Hudson River basin water and related land resources study: New York State Depart- 。 ment of Environmental Conservation, 39 p., 2 appendices.

U.S. Water Resources Council, Hydrology Commttee, 1976, Guidelines for determining flood flow frequency: U.S. Water Resources Council, Bull. 17, 35 p., 14 appendices. 
Table 1. Discharge, gage height, and elevation of 100 -year floods in the Hudson River basin

NOTES

Station number: The station number is a unique number assigned by the U.S. Geological Survey to each data-collection station. These numbers are assigned in ascending order in the downstream direction. A11 stations on a tributary entering a main stream upstream from a mainstream station have lower numbers than the main-stream station. Stations on a tributary that enters between two main-stream stations have a number between those of the two stations.

County Codes: County codes are the numeric codes of the Federal Information Processing Standards (FIPS)

$\begin{array}{clrlrl}\text { Number } & \begin{array}{l}\text { New York } \\ \text { County }\end{array} & \text { Number } & \begin{array}{l}\text { New York } \\ \text { County }\end{array} & \text { Number } & \begin{array}{c}\text { Adjacent-State } \\ \text { County }\end{array} \\ 001 & \text { Albany } & 083 & \text { Rensselaer } & \text { MA003 } & \text { Berkshire, Mass. } \\ 021 & \text { Columbia } & 087 & \text { Rockland } & \text { NJ037 } & \text { Sussex, N.J. } \\ 027 & \text { Dutchess } & 091 & \text { Saratoga } & \text { VT003 } & \text { Bennington, Vt. } \\ 031 & \text { Essex } & 093 & \text { Schenectady } & \\ 035 & \text { Fulton } & 095 & \text { Schoharie } & \\ 039 & \text { Greerie } & 1105 & \text { Sullivan } & \\ 041 & \text { Hamilton } & 111 & \text { U1ster } & \\ 043 & \text { Herkimer } & 113 & \text { Warren } & \\ 057 & \text { Montgomery } & 115 & \text { Washington } & \\ 065 & \text { Oneida } & 119 & \text { Westchester } & \\ 071 & \text { Orange. } & & & \end{array}$

(Continued on next page) 
Period of record: Periods of record are the water years (October 1 to September 30), designated by the calendar years in which they end, that were used in computation of the 100-year flood discharge. Periods of record that include a break of 1 or more years are indicated by the asterisk in the period-of-record column.

Crest-stage gages: Data from crest-stage gages (stations at which only the peak stage is recorded between inspections) used in computation of the 100-year flood discharge are indicated by the letter $P$ in the periodof-record column.

Regulation: Stations at which flow regulation can significantly affect flood levels are indicated by letter $R$ in the 100-year flood-discharge column.

Gage helght of the 100-year flood: The gage height (water-surface elevation above some arbitrary gage datum) of the 100-year flood was determined from the latest avallable stage/discharge rating curve. At many of the stations, the discharge of the 100-year flood would be greater than any measured discharge; therefore, an extension of the rating curve was required. Stations for which these extensions were not practical are indicated by a double asterisk (**) in the gageheight column.

Elevation of the 100-year flood: The elevation of the 100-year flood is the flood level, in feet above mean sea level, determined from the computed gage height and the datum (base elevation) of the gage. Stations for which 100-year flood elevations were not computed because the datum was not available are indicated by the symbol H in the elevation column.

(Continued on next page) 


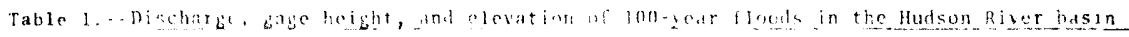

Station
riumier
Stat $10 n$ name.

C1312.j00 Hudson River below Achiont

01313500 Cedar River below Chan laken netr Indian liake

(1)3140ue lludson River at finoley mea: lindan lake Ui315t)06 Indian River near Indi in hathe

0131550 liudson kiver dt vorth l reek

01317000 seliroon River at Riverhin

01318000 Hulson River at Thurman

C] 318500 thuson River at lla liev

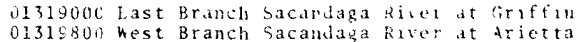

0131980 hest Branch Sacandaga Rover at

03319950 Sand Lake Out det near rinece

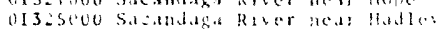

01328006 bond Creeh at luntam kasir

01328900 Fanner Brook neat Sunderland, it

01329000 batten kill at Arlingtois, it.

01329500 Fatten $k_{i} i l$ at Bat tenvili.

0133000 Clowege Creck at Weat M1lton

01330500 hayderosscras Creek near

01331500 lfoosic Rive: at Adam, Ma.
01332000 North Branch Hoosic Rivei at Vortí Adams, Ma.

01332500 lloosic River near Willambtonin, Ma.

01333000 Green River at billiamstown, M.s.

0133350 bittle loosic River at Peterhlorg

Ul333soo South Stream near Bennington, $h$ te

01333000 Paran Creek near South shaftibiry. it.

01334000 Wallomsac River near Nurth Benn

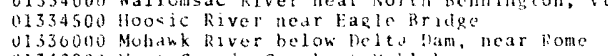

01342800 West Canada Creek at Nohlebora
01345000 West Canadd Creek at Kast Hridge

01346000 West Canadd Creek at Kast Hridge

01347000 Mchiwh River at Little Fills
01347500 East Canada Creek at Dolgeville

01348000 East Canada Creek at East Creek

0134900 otsulaso Creek at Fort Plain

0135000 Schoharie Creek at prattsilde

01351500 Schoharie creek at Burtonswlle

01354300 Plotter Kill at Ronex Corners

0135 ? 500 Mchawk Rivex at Cohoes

$01358 c 90$ Hudson River at Freen Island

01358500 Poesten Kill near Truy

01359750 Moordener $k_{2} 11$ at Castiten-on hudson

013010 ijo Kinderhook creek dt kossinai

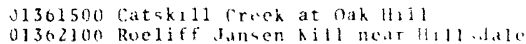

01362198 bsopus creeh at hitidatin

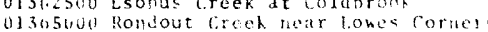

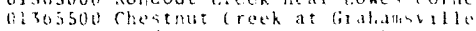

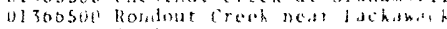

01300050 sandburg ( $r \cdots k$ it 11 lenvilt

0136750 Rondout ireek at Rosewhile

01368000 hillhild River near Unionbille

01368510 Rutgers Creek at fiarduervile

01309000 Pochuck Creek neal Pine Inland

01309500 Quaher Creeh at Florldu

01370000 Wallhill River at Pellets Tand Molntaln

01370500 Wallkill River near thili pohut

01371000 Shawangurtk $K i 11$ at $\mathrm{Pine}$ Bu

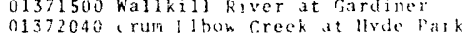

11372200 happlager ireek near ilt inton forner:

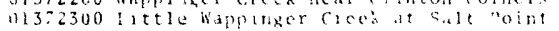

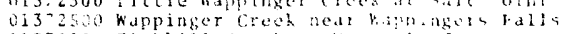
01372800 Fishki1l Creeh at Hopenely lanction 01373500 Fisink1 Creek 3t Ecacor

(1374460 South Brinch Miniaceonge Crech at

letchworth Villdge
01376500 Sam Mill River it. Yonker.

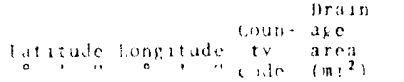

the record

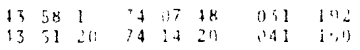

$13,11+941144$

1) $4,307: 16105$

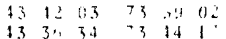

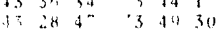

$\begin{array}{llllll}43 & 30 & 18 & 73 & 30 & 41 \\ 13 & 28 & 12 & -4 & 13 & 12\end{array}$

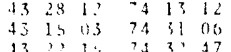

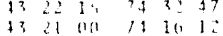

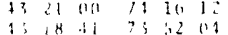

(11) 11

192675
$1931-01$

Plogiz 640

(1) 119 1911.8

$\begin{array}{lll}113 & -92 & 1908 \\ 117 & 0.29 & 1908\end{array}$

$1131533 \quad 1008219$

$091 \quad 1064 \quad 1922-75$

$\begin{array}{rrr}(14) & 114 & 193475 \\ \text { (14) } & 88.9 & 11963.75\end{array}$

(1.11 7.16 P1962 75

(11) i11 1912.75

$\begin{array}{lllllll}3 & 18 & 30 & 73 & 33 & \mathrm{Ji}\end{array}$

115

43 18 $36,73 \quad 3.304 \quad 115 \quad 11.7 \quad 1948-75 *$

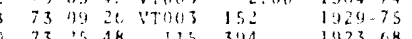

$430148 \quad 73 \quad 5548$ 001 $26.0 \quad 192360.6$

$\begin{array}{llllllllll}13 & 02 & 24 & 33 & 51 & 36 & 0191 & 90.1 & 1927-75\end{array}$

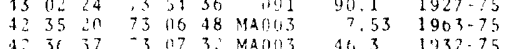

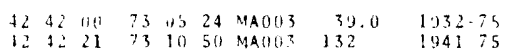

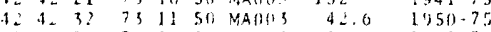

42 it $36 \quad 732624$ OBs 50.101952 .75

$42.955 \quad 73 \quad 10 \quad 14$ vT003 $\quad 7.70 \quad 51903-74$

$425813 \quad 73 \quad 11 \quad 19$ VT003 2.38 P1904-75

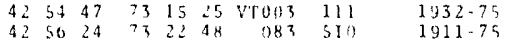

$\begin{array}{lllllllll}43 & 15 & 52 & 5 & 26 & 12 & 065 & 150 & 192875\end{array}$

$\begin{array}{lllllllll}43 & 23 & 47 & 74 & 51 & 34 & 043 & 192 & 1958-75 \\ 45 & 04 & 08 & 74 & 59 & 26 & 043 & 550 & 1921-75\end{array}$

$\begin{array}{lllllll}43 & 04 & 08 & 74 & 59 & 26\end{array}$

$\begin{array}{llllll}43 & 00 & 52 & 74 & 46 & 45 \\ 43 & 06 & 04 & 74 & 40 & 13\end{array}$

$\begin{array}{llllll}43 & 11 & 12 & 74 & 44 & 24\end{array}$

$\begin{array}{llllll}42 & 55 & 48 & 74 & 37 & 48 \\ 42 & 17 & 12 & 74 & 26 & 24\end{array}$

$\begin{array}{llllll}42 & 17 & 12 & 74 & 26 & 24 \\ 42 & 37 & 42 & 74 & 11 & 08\end{array}$

4245 on 741548

$424910 \quad 7404 \quad 211$

$\begin{array}{llllll}42 & 47 & 0 & 73 & 42 & 29 \\ 42 & 45 & 68 & 73 & 41 & 22\end{array}$

$\begin{array}{llllll}42 & 45 & 48 & 73 & 41 & 22 \\ 42 & 43 & 48 & 73 & 37 & 48\end{array}$

4232 112 33 प4 15

$4251948 \quad 734424$

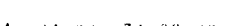

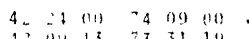

$\begin{array}{llllll}12 & 1013 & 13 & 73 & 3 ! & 10 \\ +2 & 66 & 59 & 74 & 23 & 211\end{array}$

4 in 51 iो 1 is 110

$\begin{array}{llllll}11 & 52 & 12 & 74 & 30 & 24 \\ 21 & 51 & 24 & 4 & 3 & 4\end{array}$

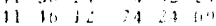

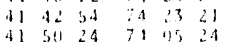

$\begin{array}{llllll}41 & 501 & 24 & 71 & 195 & 24 \\ 10 & 15 & 30 & 4 & 3 & 00\end{array}$

$\begin{array}{llllll}11 & 20 & 24 & 74 & 25 & 21 \\ 11 & 10 & 12 & 74 & 25 & 12\end{array}$

$\begin{array}{llllll}11 & 10 & 12 & 74 & 25 & 12 \\ \text { i1 } & 20 & 21 & -1 & 3 & 76\end{array}$

$\begin{array}{llllll}11 & 2 & 4 & 11 & 24 & 36 \\ 31 & 20 & 411 & 71 & 2 & 211\end{array}$

$\begin{array}{llllll}41 & 41 & 2 & 74 & 10 & 12 \\ +1 & 17 & 24 & 73 & 55 & 53\end{array}$

$+1485075050$

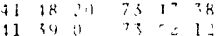

$\begin{array}{llllll}11 & 39 & 1 & 3 & 4 & 25 \\ 41 & 34 & 22 & 3 & 48 & 25 \\ 11 & 30 & 30 & 7 & 57 & 00\end{array}$

$411215 \quad 7 \div 0154$

$\begin{array}{lllllll}41 & 12 & 15 & 7 & 0 & 01 & 54 \\ 40 & 54 & 11 & 7 & 5 & 3 & 0\end{array}$

$\begin{array}{rrr}043 & 550 & 1921-75 \\ 0431348 & 1928.75\end{array}$

$\begin{array}{rrr}043 & 1348 & 1928 \cdot 75 \\ 043 & 261 & 1899-46\end{array}$

$\begin{array}{ccc}043 & 241, & 194675 \\ 057 & 59.2 & 1950.75\end{array}$

$\begin{array}{ccc}59.2 & 1950-75 \\ 339 & 536 & 1908-75 \\ 73.0 & 1924-68\end{array}$

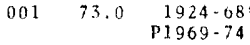

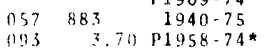

$\begin{array}{lll}083 & 89.4 & 1946-75 \\ 083 & 32.68\end{array}$

$\begin{array}{lll}183 & 89.4 & 1924-68 \\ 083 & 32.6 & \text { J958-75 }\end{array}$

$\begin{array}{lll}021 & 320 & 1029-68\end{array}$

$1961-68$
p1969-75

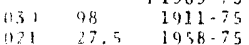

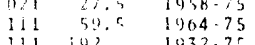

$\begin{array}{lll}111 & 149 & 1932.75 \\ 115 & 38.9 & 1937.75\end{array}$

li, 20.9 log 1937.5

lif lin, lo3, 50

11! 5n.7 lis 75

$\begin{array}{lll}111 & 360 & 1926-54 \\ 137 & 140, & 1938-75\end{array}$

$\begin{array}{lll}71 & 59.7 & 1944-68 \\ 811 & 58.0 & 1338-75\end{array}$

$\begin{array}{lll}071 & 9.74 & 103875 \\ 071 & 3 \times 5 & 1320-68\end{array}$

$071 \quad 3 \times 5$

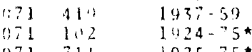

m.7 18.t $\begin{array}{r}1925-75 \\ 1960-62\end{array}$

1.2 $42.4 \begin{array}{r}196,75-75 \\ 1950.75\end{array}$

$\begin{array}{lll}127 & 92.4 & 1956-75 \\ 127 & 32.9 & 1956-75 \\ 127 & 182 & 1929-75 \\ 027 & 5-7 & 1900-75\end{array}$

$\begin{array}{lll}02^{-} & 5.7 & 1904-75 \\ 1127 & 18 \% & 1945-68\end{array}$

198 $5.83 \quad r 1960-75$

(1) 3.3.71) P1958-74* R4 220

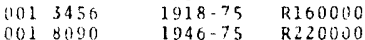

11000
1900

1900
29000

Lue Year flood

ischarye height Elevation

(ft) (ft) . sea level)

$\begin{array}{lll}7700 & 12.3 & 1562.7 \\ 12100 & 13.7 & 1553.7\end{array}$

10000

83400
$R 2000$

PIxniag
$370+90$

42000

110000
3400

400
320140

35000

1706

130
9500

21000

4400

1200
42610

**"

$\begin{array}{rl}7.7 & 1611.9 \\ 12.5 & 1000.0\end{array}$

$14.3 \quad 713.6$

$20.9 \quad 584.9$

$14.4 \quad 1268.7$

$\begin{array}{rr}4.2 & 1691.6 \\ 10.7 & 892.0\end{array}$

a*

9.4

117.2

11.7

7.9
10.4
5.8
9.1

608.9

368.8
415.5

415.5
368.5

$8 \overrightarrow{3} 7.1$

$\begin{array}{lll}11000 & 13.0 & 833.5 \\ 14000 & 14.2 & ++\end{array}$

4000

5400
8200
200
340

340
11000

11000

43090
$\mathrm{R} 8000$

14300

R 19000
R 28000

16000

18000

74000

7200

6.2
11.4

17

13.0

18.8
18.5
11.4

11.4
7.8

19.0
14.3

14.3
8.4

11.4

598.8

$+$

++
++
374.2
485.5

485.5

1400.6

446.8
327.8

327.8
764.3

344.1
314.6

921.5

8.8516 .8

516.8

73.5

334.1

334.1
102.8

12.6
4.1
19.3
$k \pi$

45.1

16000

200010

82000

11000

84011
270015

$\therefore 100$

32000

5000
7000

3900

1400

12000
11000

10000

31000

8200

2 2500

200010
1600

13000

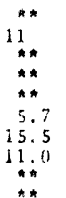

$\because+$

$* \quad \therefore$

12.0

9.4
7.0

24.5
12.4

18.1
18.5

1100

16.0
8.4

8.4
20.4
10.4

10.4
15.9

251,1

134.8
239.9

** 6

98.6 